\title{
Focus on therapy of the Chapter IV headaches provoked by exertional factors: primary cough headache, primary exertional headache and primary headache associated with sexual activity
}

\author{
Marta Allena • Paolo Rossi - Cristina Tassorelli • \\ Enrico Ferrante $\cdot$ Carlo Lisotto $\cdot$ Giuseppe Nappi
}

Received: 4 July 2010/Accepted: 15 September 2010/Published online: 2 October 2010

(C) Springer-Verlag 2010

\begin{abstract}
Primary cough headache, primary exertional headache and primary headache associated with sexual activity are distinct entities, even though they share several features: acute onset, the absence of structural brain disease and exertional factors as precipitating events. In this short review, we illustrate the possible treatment strategies on the basis of information collected from a systematic analysis of the international literature.
\end{abstract}

Keywords Other primary headaches · Cough headache . Exertional headache . Headache associated with sexual activity $\cdot$ Treatment

\section{Background}

The second edition of the International Classification of Headache Disorders (ICHD-II) [1] includes in Chapter IV,

\author{
M. Allena $\cdot$ C. Tassorelli $(\bowtie) \cdot$ G. Nappi \\ Headache Science Centre, IRCCS “C. Mondino National \\ Neurological Institute" Foundation, Pavia, Italy \\ e-mail: cristina.tassorelli@mondino.it \\ P. Rossi \\ Headache Clinic INI Grottaferrata, Rome, Italy \\ C. Tassorelli · G. Nappi \\ University Centre for Adaptive Disorders and Headache \\ (UCADH), University of Pavia, Pavia, Italy \\ E. Ferrante \\ Department of Neuroscience, Niguarda Ca' Granda Hospital, \\ Milan, Italy \\ C. Lisotto \\ Department of Neuroscience, Headache Centre, \\ S. Vito al Tagliamento Hospital, Pordenone, Italy
}

among the "Other Primary Headaches", three forms that share some clinical characteristics and have similar underlying pathophysiological mechanisms: primary cough headache, primary exertional headache and primary headache associated with sexual activity.

These three types of headache have been known and described in the medical literature since the 1930s [2]. Initially, a headache induced by coughing or by exertion was considered as an ominous symptom and it was not until after 1956, when cases of both primary and secondary headaches provoked by coughing and not precipitated by physical exercise were described [3]. It became clear that these three disorders are distinct entities, even though they share several features: acute onset, the absence of structural brain disease and exertional factors as precipitating events.

In the following paragraphs, we review treatment strategies for primary cough headache, primary exertional headache and primary headache associated with sexual activity, on the basis of information collected from a systematic analysis of the international literature. We conducted a baseline literature search covering the period 1932-2009, employing available electronic databases (National Library of Medicine, National Institute of Health, Embase) and the following medical search terms: cough, exertional, exercise, strain, orgasmic, sex and headache. Whenever available, book chapters were also consulted and considered.

\section{Primary cough headache}

The benign interplay between headache and coughing (i.e. in the absence of any intracranial lesions) is actually wellknown. Primary cough headache was first described in 1932 by Tinel [2], who reported four patients with headache pain 
triggered by a single Valsava manoeuvre or by a very short series of Valsava-type maneouvres (i.e. by sneezing, coughing or straining during defaecation). For many years, cough headache like exertional and sexual headaches were considered as an alarm signal; until Symonds [3] in 1956 and Rooke [4], a decade later, recognised some benign or primary forms of these disorders.

Primary cough headache is considered as a rare disorder, with a lifetime prevalence of $1 \%$ [5]. Typically, onset is after the fourth decade of life and in some cases, it follows chest diseases. The clinical picture of this primary headache is very characteristic, and it consists of bilateral pain (occasionally unilateral) that arises suddenly after the precipitating event and lasts for between a few seconds and $30 \mathrm{~min}$ [1]; it is not associated with any neurovegetative or local autonomic symptoms. Some patients complain of a dull, persistent pain between attacks. Unilateral benign cough headache has also been reported in association with chronic paroxysmal hemicrania [6].

For this headache to be considered a benign condition, the ICHD-II criteria [1] specify that structural lesions, especially in the cerebral posterior fossa, must be ruled out by diagnostic neuroimaging. Indeed, cough headache is symptomatic in about $40 \%$ of cases and (differently from what is found in exertional and in sexual headache) in the largest reported series, the symptomatic forms were even more frequent than the benign ones [7]. The condition most frequently associated with cough headache is a type I Chiari's malformation [3, 7] while the headache is rarely secondary to space-occupying lesions in the posterior fossa, or to carotid, vertebrobasilar or other intracranial vessel pathologies [8, 9].

While the pathophysiology of secondary cough headache is quite well understood, the aetiology of the benign form is more obscure. It seems feasible that the headache derives from a temporal impaction of cerebral structures situated below the foramen magnum as a result of the transient increase in intracranial pressure following increases, during coughing, in intrathoracic and intraabdominal pressure. It is possible that in individuals affected by this headache, the increased intracranial pressure normally caused by coughing may combine with a lower pain threshold. Wang and colleagues [10] postulated that the benign form is the result of cerebrospinal hypervolaemia which explains the response to treatments that decrease intracranial pressure, i.e. acetazolamide, indomethacin and lumbar puncture (see later).

Recently, the same group of researchers investigated the morphometric characteristics of the posterior cranial fossa by means of MRI in 18 patients with primary cough headache compared with age- and sex-matched controls [11]. The authors reported evidence of posterior fossa overcrowding in the headache patients, suggesting that this may be a contributing factor in the pathogenesis of benign cough headache, probably causing an "relative" obstruction in the cerebrospinal pathway during coughing.

Treatment of primary cough headache

In view of the short duration of the pain after the precipitating event, the most important therapeutic approach is prevention. In 1981, in a double-blind study involving two patients with cough headache, indomethacin, given prophylactically with the dosage of $150 \mathrm{mg}$ per day, emerged as the first effective drug [12]. The efficacy of indomethacin was confirmed in subsequent studies at therapeutic doses ranging 50-250 mg daily, usually in association with a proton pump inhibitor in patients requiring long-term treatment $[13,14]$.

Case reports of patients responsive to acetazolamide [10] and methysergide $[15,16]$ have been published, suggesting that these drugs have a similar mechanism of action to indomethacin (i.e. reduction of intracranial pressure).

Some authors have reported benefit following lumbar puncture in patients with cough headache [3, 4, 13]. Raskin [13] performed lumbar punctures in 14 patients: 6 had complete relief while 8 did not respond to this treatment; later on, 6 patients obtained improvement with indomethacin. Naproxen [6, 14], ergonovine, IV dihydroergotamine and phenelzine [14] have also been reported to be effective. Propranolol caused remission of the disease in one case [15].

Recently, Medrano and colleagues [17] described three patients with primary cough headache who were intolerant to indomethacin. They were thus treated with topiramate which induced a significant improvement in one patient and a partial response in the other two.

Further reports and studies are needed to clarify the effective therapeutic options in patients with cough headache.

\section{Primary exertional headache}

Primary exertional headache, as defined by the ICHD-II criteria [1], is a pulsating headache lasting from $5 \mathrm{~min}$ to $48 \mathrm{~h}$, brought on by and occurring only during or after physical exertion, and not attributed to any other disorder. Unlike cough headache, where pain can be triggered by short-lasting trains of efforts (i.e. Valsalva-like manoeuvres), in the case of exertional headache, the precipitating factor is usually sustained by physically strenuous exercise $[7,18]$.

Even though a large proportion of individuals with primary exertional headache also suffer from migraine- $46 \%$ in the Vågå study [18] and 54.9\% in a survey of adolescents [19] — and exertional headache may have migrainous 
features, exertional headache nevertheless seems to be essentially different from migraine [20].

For a long time, this headache was considered a relatively uncommon disorder. In two general population studies that applied the criteria of the first edition of the International Headache Society's ICHD-II of 1988 [21], one in Portugal and other in Denmark, the prevalence of exertional headache was estimated to be 0.2 and $1 \%$, respectively [22, 23]. More recently, in the Vågå study [18] of headache epidemiology, Sjaastad and Bakketeig [19] reported a $12.9 \%$ lifetime prevalence of exertional headache with a slight but significant female preponderance (F:M ratio 1.38). This marked discrepancy can hardly be explained by chance alone and probably reflects differences in the study designs or questioning techniques used. In a community study of students aged 13-15 years, conducted in Taiwan using the ICHD-II criteria, Chen et al. reported a lifetime prevalence of $30.4 \%$, decreasing with age, with girls having a higher prevalence of exertional headache than boys (36.3 vs. $24.4 \%, p<0.001)$. These findings suggest that exertional headache is common among adolescents, and indeed, in Sjaastad's studies $[18,20]$ as well as in Pascual's [7] clinical series, exertional headache seems to be a juvenile headache. Interestingly, following the onset of benign exertional headache, the attacks, occurring with a frequency of between one per day and one every 2 months, recurred over a very variable period of time usually lasting $<20$ years. The majority $(85.7 \%)$ of Chen et al.'s adolescents reported that exertional headache occurred occasionally [19]. In the Vågå study, approximately $40 \%$ of the patients experienced exertional headache only during circumscribed periods of their life which suggests a role played by predisposing factors that vary intraindividually [20].

The pathophysiological mechanisms underlying this form of headache are unknown. Most investigators believe that these headaches are vascular in origin, hypothesising that a venous or an arterial distension, secondary to physical exercise, is the pain-inducing mechanism [24, 25].

The recent finding that patients with primary exertional headache have a significantly higher prevalence of internal jugular vein valve incompetence ( $70 \%$ of patients vs. $20 \%$ of controls) suggests that intracranial venous congestion caused by retrograde jugular venous flow might play a role in the pathophysiology of this disorder [26].

Treatment of primary exertional headache

The treatment of exertional headache has not been adequately studied and current evidence is not sufficient to allow the development of treatment guidelines. However, because of the nature of the disease, specific treatment is rarely called for. In the Taiwanese study [19], only $13.8 \%$ of the students, mainly those with migrainous features, used painkillers to treat their exertional headache. Indeed, this is a benign headache with a self-limiting course and the intensity of the pain is moderate [18]. It usually takes only a few episodes for the patient to understand what factors precipitate single episodes and to learn (often without consulting a physician) to avoid the physical exertion that triggers the pain.

This behavioural measure of avoiding or reducing the precipitating factor is considered the preventive treatment of choice for exertional headache [27]. When exertion is unavoidable, indomethacin three times daily is the most frequently recommended approach, even though no controlled trials have been done to support this recommendation [28, 29].

Several other drug treatments have been reported to be effective in small case series. In a retrospective case series including 16 patients with benign exertional headache, Pascual et al. [7] reported that ergotamine (unspecified dose) taken immediately before exercise was subjectively effective in four patients. Propranolol, given to five patients, was effective in one case possibly effective in three cases and ineffective in the remaining patient who instead had clear benefits with indomethacin. Flunarizine was tried in two patients and was effective in one. In a more recent case series, Pascual and colleagues [30], reported that five patients need preventive treatment, selected from among nine subjects diagnosed with primary exertional headache, responded to nadolol or propranolol $1-2 \mathrm{mg} / \mathrm{kg}$ per day.

Further studies focusing on the treatment issues in this headache are necessary.

\section{Primary headache associated with sexual activity}

Since the original description in the 1970s [31-33], benign sexual headache has become a well-defined entity. The entity "Headache associated with sexual activity" featured in the first edition of ICHD-II of 1988, in the Chapter "Miscellaneous headaches unassociated with structural lesion" [21]. In that classification, this form of headache was subdivided into three subtypes on the basis of the pain features and the presumed clinical pathophysiological mechanisms: "dull type" (a muscle contraction type of headache with bilateral pressure pain that gradually increases with sexual excitement), "explosive type" (a vascular type of headache with abrupt and throbbing pain occurring at the moment of orgasm) and "postural type" (a holocephalic, positional headache developing after coitus, with many clinical features consistent with low cerebrospinal fluid pressure headache and presumed to be related to a dural tear occurring during intercourse). In ICHD-II [1], this headache was included in Chapter IV 
under the heading "Primary headache associated with sexual activity" and subdivided, according to the timing of its onset in relation to sexual activity, into pre-orgasmic headache (code 4.4.1) and orgasmic headache (code 4.4.2), with these two subtypes corresponding to the two first types in the 1988 classification. The third type in the 1988 classification was instead moved to the section of secondary headache disorders, where it was denoted "Headache attributed to spontaneous (or idiopathic) low CSF pressure" (code 7.2.3).

The most common type of primary headache associated with sexual activity is the explosive one (about 70\%), now called orgasmic headache. However, because of its sudden onset and its rapid ascent to maximal severity, features that may suggest a symptomatic headache, it is also the one most likely to be considered as a "warning signal". At first onset of orgasmic headache it is mandatory to exclude secondary headache conditions (such as subarachnoid haemorrhage and arterial dissection and, more rarely, intracranial masses), even though they are rare. Studies have shown that subarachnoid haemorrhage precipitated by coitus occurs in 4-12\% of all cases with saccular aneurysm and in $4.1 \%$ of patients with arterio-venous malformations $[34,35]$.

The prevalence of primary headache associated with sexual activity in the general population is not well-defined and it is difficult to determine. This is an uncommon, but probably under-diagnosed and under-reported headache, because patients often feel embarrassed to give details about their intimate sexual activities. In the only population-based epidemiological study conducted, Rasmussen and Olesen [5] found a prevalence of about $1 \%$ in a 25- to 64-year-old population, similar to that of the other two primary headaches provoked by exertional factors: cough headache and exertional headache. In three headache clinic-based studies, patients fulfilling the criteria for headache attributed to sexual intercourse ranged from 0.2 to $1.3 \%$ [36-38], while a recent Turkish population-based study reported a prevalence of $1.6 \%$, with an age at onset and a gender distribution similar to those given in other reports [39].

This primary headache disorder has a clear male preponderance $(4: 1)$, the mean age at onset showing it to affect young up to people in the second and third decades of life. In the German study by Frese and colleagues [38], two peaks in relation to age at onset were noted, an early peak between 20 and 24 years and a rather late one, between 35 and 44 years, but the significance of this observation is unclear.

The duration of the headache pain also varies from individual to individual. It may last just a few minutes, or in more severe cases, between a couple of hours and a day. The common feature in all cases is intense pain in the first
5-15 min, thereafter gradually subsiding. The duration of the pain is longer in headache with onset during orgasm than in pre-orgasmic headache (4 vs. $1 \mathrm{~h}$ ). The location of the pain is bilateral in $75 \%$ of patients. Nausea and phono/ photophobia are uncommon. The literature also reports the case of one woman who complained of migraine with aura attacks triggered by orgasm [40].

The occurrence of primary headache associated with sexual activity is not dependent on specific sexual habits, although it is most frequent during sexual activity with the usual partner $(94 \%)$ or during masturbation $(35 \%)$ [38, 41]. In some cases, this headache type occurred only during masturbation [38, 42].

Headache associated with sexual activity frequently occurs in patients with other types of primary headache, such as migraine (with a prevalence ranging from 25 to $47 \%)$, exertional headache $(31-40 \%)$ and, more rarely, cough headache $[7,38,43,44]$. These associations were not completely confirmed in an Indian population study, in which few patients with sexual headache had associated migraine or other primary headaches and none ever experienced exertional headache [41]. However, in a recent case-control study of migraine patients investigating the comorbidity of migraine and sexual headache, a possible bilateral and not only unilateral association between these two primary headache forms was detected [45].

Headache associated with sexual activity has an unpredictable course, sometimes occurring at regular intervals, and sometimes occurring sporadically. Ostergaard and Kraft [46] reported the clinical course of 26 patients followed up for up to 14 years. Some patients presented only an isolated episode or a single cluster of sexual headaches, while most of them had several episodes without a clearly defined periodicity or regularity. Silbert and colleagues [43] noted that in the majority of their patients headache related to sexual activity appeared in bouts of some weeks or months and disappeared without any treatment. A recent study confirmed the favourable prognosis of this condition, recording-remission rates of $69 \%$ during a 3-year observation period, and two different temporal patterns of the disorder: an episodic course with remitting bouts (most patients) and a chronic course (approximately a quarter of patients), as observed in other primary headaches such as cluster headache and paroxysmal hemicrania [47].

The pathophysiology of this headache remains unknown and often speculative. Some years ago, Lance suggested that excessive muscle contraction (selectively in neck and cranial muscles) during sexual activity is responsible for the pre-orgasmic type, while a vascular origin has been proposed for the orgasmic type (rapid increases in blood pressure and heart rate during the orgasmic phase), although in both cases these simple explanations appear incomplete [2]. 
There is at least one report of familial sexual headache (four sisters suffering from orgasmic headache with "vascular features") [48], but this does not seem enough to support a genetic basis of the disorder.

Treatment of primary headache associated with sexual activity

No meta-analyses are available on the treatment of this type of headache. Randomised trials and case reports showed good efficacy of short-term prophylaxis with indomethacin 25-100 mg 1-2 h before intercourse in patients with regular and predictable sexual activity [7, 43, 49]; other nonsteroidal anti-inflammatory drugs (ibuprofen and diclofenac), like analgesics (ibuprofen, paracetamol, diclofenac and ASA), given after the onset of headache, are of limited or no value.

A single case study by Evans [50] reported benefit with naratriptan $2.5 \mathrm{mg} 2 \mathrm{~h}$ before sexual activity, and recently, Frese and colleagues also reported, in some patients, a positive response to triptans taken prior to the sexual intercourse [47, 51]. Therefore, it seems possible to propose triptans as a possible alternative for patients not tolerating indomethacin.

If the problem persists or in patients with longer lasting bouts, beta-blockers (propranolol 120-240 mg per day, metopropolol 100-200 mg per day and diltiazem $180 \mathrm{mg}$ per day) are recommended as a prophylactic therapy [7, 43, 49, 52]. Lamotrigine, $100 \mathrm{mg}$ daily, was used in the reported case of migraine with aura occurring after sexual intercourse [40]. Recently, a Turkish patient with orgasmic headache responded to single greater occipital nerve injection with steroid and local anaesthetic [53], but since this headache can remit spontaneously a natural cessation cannot be excluded.

Once secondary causes have been excluded, people who suffer from this condition should be reassured that it is rarely life-threatening. In most cases, the problem will disappear on its own, because it is a benign disorder often with a selflimiting course. Patients should also be informed that they are at higher risk of incidence when tired, stressed, or engaging in sexual intercourse several times in rapid succession. There are also some behavioural measures that could be beneficial in sexual headache, such as changing position, keeping the neck lower than the trunk, working the lower limbs less, assuming a passive sexual role [32] and losing weight.

\section{Conflict of interest None.}

\section{References}

1. Headache Classification Subcommittee of the International Headache Society (2004) The International Classification of Headache Disorders, 2nd edn. Cephalalgia 24(Suppl 1):1-160
2. Tinel J (1932) La cephalée à l'éffort. Syndrome de distension doloureuse des veines intracraniennes. Medicine (Paris) 13:113-118

3. Symonds C (1956) Cough headache. Brain 79(4):557-568

4. Rooke ED (1968) Benign exertional headache. Med Clin North Am 52:801-808

5. Rasmussen BK, Olesen J (1992) Symptomatic and nonsymptomatic headaches in a general population. Neurology 42: $1225-1231$

6. Mateo I, Pascual J (1999) Coexistence of chronic paroxysmal hemicrania and benign cough headache. Headache 39:437-438

7. Pascual J, Iglesias F, Oterino A et al (1996) Cough, exertional and sexual headaches. An analysis of 72 benign and symptomatic cases. Neurology 46:1520-1524

8. Britton TC, Guiloff RJ (1988) Carotid artery disease presenting as cough headache. Lancet 1:1406-1407

9. Smith WS, Messing RO (1993) Cerebral aneurysm presenting as cough headache. Headache 33:203-204

10. Wang SJ, Fuh JL, Lu SR (2000) Benign cough headache is responsive to acetazolamide. Neurology 55:149-150

11. Chen YY, Lirng JF, Fuh JL et al (2004) Primary cough headache is associated with posterior fossa crowdedness: a morphometric MRI study. Cephalalgia 24:694-699

12. Mathew NT (1981) Indomethacin responsive headache syndromes. Headache 21:147-150

13. Raskin NH (1995) The cough headache syndrome: treatment. Neurology 45:1784

14. Raskin NH (1988) The indomethacin-responsive syndromes. In: Raskin NH (ed) Headache, 2nd edn. Churchill Livingstone, New York, pp 255-268

15. Calandre L, Hernandez-Lain A, Lopez-Valdes E (1996) Benign Valsalva's maneuver-related headache: an MRI study of six cases. Headache 36:251-253

16. Bahra A, Goadsby PJ (1998) Cough headache responsive to methysergide. Cephalalgia 18:495-496

17. Medrano V, Mallada J, Sempere AP, Fernández S, Piqueras L (2005) Primary cough headache responsive to topiramate. Cephalalgia 25:627-628

18. Sjaastad O, Bakketeig LS (2002) Exertional headache.I. Vågå study of headache epidemiology. Cephalalgia 22:784-790

19. Chen SP, Fuh JL, Lu SR, Wang SJ (2009) Exertional headachea survey of 1963 adolescents. Cephalalgia 29:401-407

20. Sjaastad O, Bakketeig LS (2003) Exertional headache-II.Clinical features Vågå study of headache epidemiology. Cephalalgia 23:803-807

21. Headache Classification Committee of the International Headache Society (1988) Classification and diagnostic criteria for headache disorders, cranial neuralgias and facial pain. Cephalalgia 8(suppl 7):1-89

22. Monteiro JF (1985) Cefaleias. Estudio epidemiologico e clinic de una populacao urbana. Thesis. Porto

23. Rasmussen BK (1994) Epidemiology of headache. Thesis. Kobenhavns Universitet, Copenhagen

24. McCrory P (1997) Recognizing exercise-induced headache. Phys Sportsmed 25:33-43

25. Buzzi MG, Formisano R, Colonnese C et al (2003) Chiari-associated exertional, cough and sneeze headache responsive to medical therapy. Headache 43:404-406

26. Doepp F, Valdueza JM, Schreiber SJ (2008) Incompetence of internal jugular valve in patients with primary exertional headache: a risk factor? Cephalalgia 28:182-185

27. Imperato J, Burstein J, Edlow JA (2003) Benign exertional headache. Ann Emerg Med 41:98-103

28. Diamond S (1982) Prolonged benign exertional headache: its clinical characteristics and response to indomethacin. Headache 22:96-98 
29. Dodick DW (2004) Indomethacin-responsive headache syndromes. Curr Pain Headache Rep 8:19-26

30. Pascual J, González-Mandly A, Martín R et al (2008) Headaches precipitated by cough, prolonged exercise or sexual activity: a prospective etiological and clinical study. J Headache Pain 9:259-266

31. Kríz K (1970) Coitus as a factor in the pathogenesis of neurologic complications. Cesk Neurol Neurochir 33:162-167

32. Lance JW (1976) Headaches related to sexual activity. J Neurol Neurosurg Psychiatr 39:1226-1230

33. Paulson GW, Klawans HL (1974) Benign orgasmic cephalgia. Headache 13:181-187

34. Locksley HB (1969) Natural history of subarachnoid hemorrhage, intracranial aneurysm and arteriovenous malformations. In: Sahs $\mathrm{Al}$, Perret GE, Locksley HB, Nishioka H (eds) Intracranial aneurysms and subarachnoid hemorrhage. Lippincott, Philadelphia, pp 37-57

35. Lundberg PO, Osterman PO (1974) The benign and malignant forms of orgasmic cephalgia. Headache 14:164-165

36. Kraft M (1979) Benign coital cephalgia. Violent headache developing during sexual intercourse. Ugeskr Laeger 141: 2454-2455

37. Nick J, Bakouche P (1980) Les cephalees declenchees par l'acte sexual. Sem Hop 56:621-628

38. Frese A, Eikermann A, Frese K et al (2003) Headache associated with sexual activity: demography, clinical features, and comorbidity. Neurology 61:796-800

39. Tugba T, Serap U, Esra O et al (2008) Features of stabbing, cough, exertional and sexual headache in a Turkish population of headache patients. J Clin Neurosci 15:774-777

40. D'Andrea G, Granella F, Verdelli F (2002) Migraine with aura triggered by orgasm. Cephalalgia 22:485-486

41. Chakravarty A (2006) Primary headaches associated with sexual activity-some observations in Indian patients. Cephalalgia 26:202-207

42. Vincent FM (1982) Benign masturbatory cephalalgia. Arch Neurol 39:673
43. Silbert PL, Edis RH, Stewart-Wynne EG et al (1991) Benign vascular headaches and exertional headache: interrelationships and long term prognosis. J Neurol Neurosurg Psychiatr 54:417-421

44. Evers S, Lance JW (2006) Primary headache attributed to sexual activity. In: Olesen J, Goadsby PJ, Ramadan NM et al (eds) The Headaches, 3rd edn. Lippincott Williams \& Wilkins, Philadelphia, pp 841-845

45. Biehl K, Evers S, Frese A (2007) Comorbidity of migraine and headache associated with sexual activity. Cephalalgia 27:1271-1273

46. Ostergaard JR, Kraft M (1992) Benign coital headache. Cephalalgia 12:353-355

47. Frese A, Rahamann A, Gregor N et al (2007) Headache associated with sexual activity: prognosis and treatment options. Cephalalgia 27:1265-1270

48. Johns DR (1986) Benign sexual headache within a family. Arch Neurol 43:1158-1160

49. Frese A, Frese K, Schwaag S et al (2004) Prophylactic treatment and course of the disease in headache associated with sexual activity. In: Olesen J et al (eds) Preventative Pharmacotherapy of Headache Disorders. Oxford University Press, Oxford, pp 50-54

50. Evans RW, Pascual J (2000) Orgasmic headaches: clinical features, diagnosis and management. Headache 40:491-494

51. Frese A, Gantenbrein A, Marziniak M et al (2006) Triptans in orgasmic headache. Cephalalgia 26:1458-1461

52. Porter M, Jankovic J (1981) Benign coital cephalalgia. Differential diagnosis and treatment. Arch Neurol 38:710-712

53. Selekler M, Kutlu A, Dundar G (2009) Orgasmic headache responsive to greater occipital nerve blockade. Headache 49:130-147 